\title{
Comparison of the Convergent and Divergent Runners for a Low Head Hydraulic Turbine
}

\author{
Zbigniew Krzemianowski ${ }^{1}$ and Romuald Puzyrewski ${ }^{2}$ \\ 1. Department of Hydropower, The Szewalski Institute of Fluid-Flow Machinery, Polish Academy of Sciences, Fiszera 14, Gdansk \\ 80-231, Poland \\ 2. Department of Energy and Industrial Apparatus, Mechanical Faculty, Gdansk University of Technology, Narutowicza 11/12, \\ Gdansk 80-233, Poland
}

Received: September 29, 2015 / Accepted: October 30, 2015 / Published: December 31, 2015.

\begin{abstract}
The paper presents a method of the runner blades design for simple case of hydraulic turbine. Two differently shaped channels of meridional cross section were examined. The quantitative evaluation was performed by means of $3 \mathrm{D}$ algorithm. It has been found that, divergent runner prevails convergent from dissipation point of view. Although, the influence of draft tube has not been analyzed, the presented method is important for the designers of low head hydraulic turbines which are not the matter of standardization due to variety of environmental conditions.
\end{abstract}

Key words: Hydraulic machinery design, inverse problem, 2D model.

\section{Introduction}

The paper is devoted to the design of runner blade cascade supported by 2D and 3D computation models, for low head hydraulic turbine. The necessity of developing the method of design comes from the fact that, planned low head hydraulic turbines are not the objects for standardization due to variety of environment, in which such installations are foreseen. Also from theoretical point of view, the problem is interesting because it insists to develop the method of finding the geometry of designed blades. Here, the attention is focused on two models. Model 2D is presented in the version of the so-called inverse problem in which the boundary conditions do not contain geometry of blades. The model allows creating such geometry. If geometry is determined from 2D model, then 3D computation may be the next step to check the flow field. The aim of the analysis was focused on finding the geometry where minimum

Corresponding author: Zbigniew Krzemianowski, $\mathrm{PhD}$, research associate, research fields: hydraulic turbines designing and analyzing, hydraulic turbines measurement. losses are expected. Besides, the influence of turbine meridional shape in the domain of runner in two versions: convergent and divergent was investigated. It was also interesting to find the answer whether the function of diffuser behind the runner can be partly overtaken by contour as it is in divergent runner.

Here, the attention is focused on two differently shaped runners. Convergent shape of runner leads to an increase of the load of draft tube behind the runner if the outlet of draft tube is imposed. One has to be aware of that, the increase of draft tube load may change the final conclusions.

The main task of the presented paper is the method of the runner blade design. For 3D computation, commercial code ANSYS/Fluent 15 was applied. 2D model used to calculations was described in Ref. [1].

\section{Geometrical Boundary Conditions}

The geometry of two contours of the flow field discussed here is shown in Fig. 1.

In both cases, the family of lines (they can be treated as a wishful order of stream surfaces flowing by a 


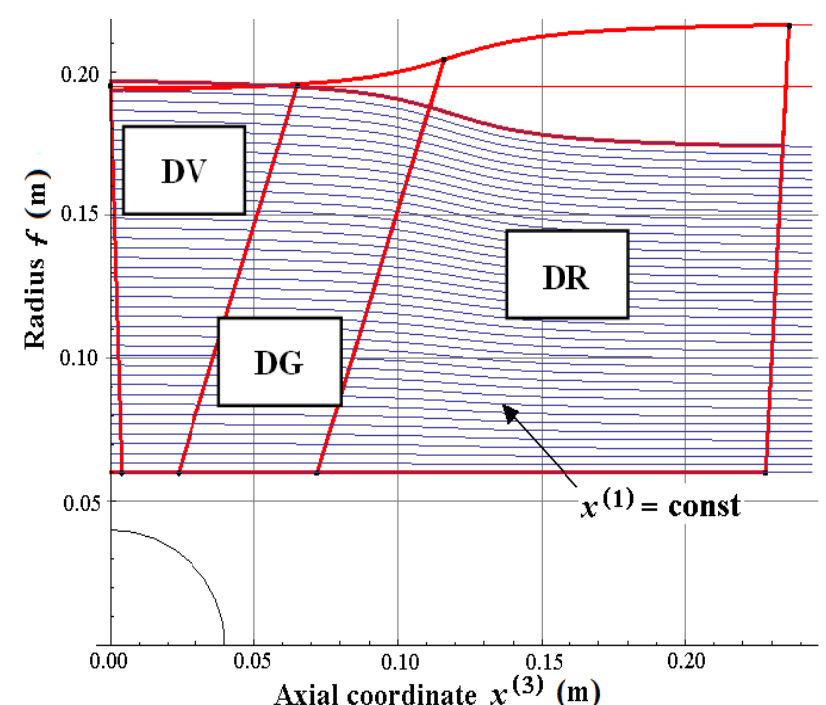

(a)

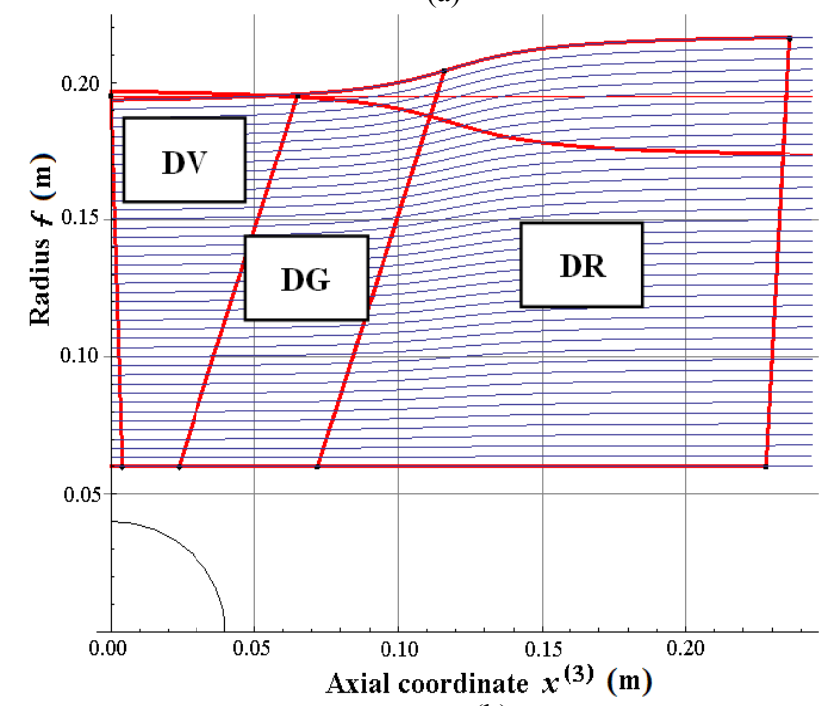

(b)

Fig. 1 Two views of flow domain: (a) convergent runner and (b) divergent runner.

meridional plane) shown in Fig. 1, covers the domains of guide vanes (DV), gap (DG) and runner (DR). These lines were used in non-orthogonal system of coordinates describing the outer border denoted as $x^{(1)}=0$ and internal border denoted by $x^{(1)}=1$. The angular coordinate is denoted by $x^{(2)}$ and axial coordinate by $x^{(3)}$.

The outer line for converging domain is given as the radius function $f$ dependent on axial coordinate $x^{(3)}$ :

$$
\begin{gathered}
f_{\text {outer con }}= \\
0.1854-0.008433 \operatorname{ArcTan}\left(\frac{x^{(3)}}{0.0268}-4.421\right)
\end{gathered}
$$

and for diverging domain:

$$
\begin{gathered}
f_{\text {outer div }}= \\
0.205+0.008433 \operatorname{ArcTan}\left(\frac{x^{(3)}}{0.0268}-4.421\right)
\end{gathered}
$$

The inner line (hub) in both cases is equal to: $f_{\text {inner }}=$ $0.06 \mathrm{~m}$. The family of lines is given by the function of $x^{(1)}$ in the form:

$$
f=f_{\text {outer }}+\left(f_{\text {inner }}-f_{\text {outer }}\right) x^{(1)}
$$

The choice of these function types is based on intuition and experience. These functions represent quantitatively factor of a convergence and divergence. In authors' opinion, this motivation is sufficient for the analysis of a convergence and divergence upon the flow field of turbine stage.

The positions of leading and trailing edges of runner blades were also chosen intuitively.

The number of blades in runner domain belongs to the geometrical boundary conditions. According to the experience, it was taken into account three or four blades in the runner domain. The influence of number of blades was investigated by means 3D model.

\section{Boundary Conditions for Flow Parameters}

In front of runner cascade, the distributions of flow parameters were chosen as it follows from previous computation of guide vane domain. The distributions of the axial and tangential velocity components in front of runner cascade are shown in Fig. 2. The axial velocity distribution in the chosen example of geometry gives the mass flow rate $m=235 \mathrm{~kg} / \mathrm{s}$.

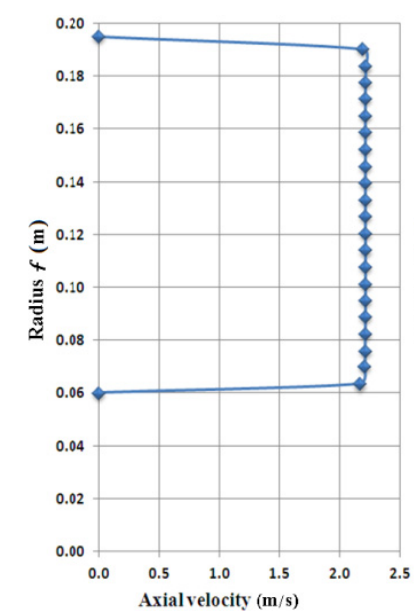

(a)

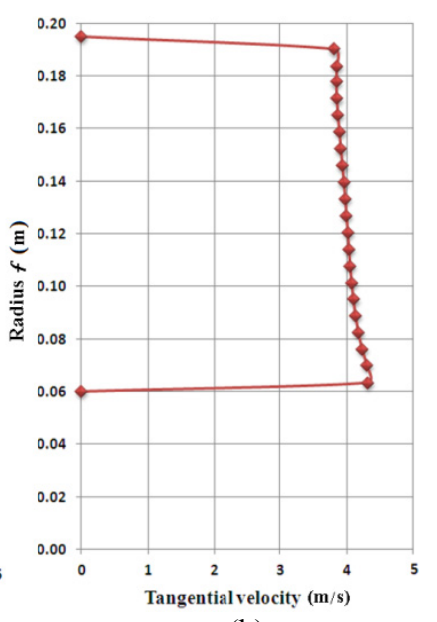

(b)
Fig. 2 Distribution of axial and tangential velocity components at inlet to the runner domain. 
Model 3D gives the pressure field from the solution of momentum equation in respect to the reference point, where the value of pressure is given. The reference for pressure level was set at the outlet to be equal zero.

For 2D inverse model, it was necessary to assume at outer contour line of runner domain distribution of pressure from inlet to outlet. The pressure distribution was given as the function of three parameters:

$$
\begin{gathered}
p\left(\overline{x^{(3)}}, p_{\text {outlet }}, n_{b}\right)=p_{\text {inlet }}+ \\
\left(p_{\text {outlet }}-p_{\text {inlet }}\right)\left(\overline{x^{(3)}}\right)^{n_{b}}
\end{gathered}
$$

where, $\overline{x^{(3)}}$ is non-dimensional coordinate: $\overline{x^{(3)}}=$ $\frac{x^{(3)}-x_{\text {inlet }}^{(3)}}{x_{\text {outlet }}^{(3)}-x_{\text {inlet }}^{(3)}} ; p_{\text {inlet }}$ is inlet pressure; $p_{\text {outlet }}$ is pressure in trailing point at outer border of runner and exponent $n_{b}$ of non-dimensional coordinate $\overline{x^{(3)}}$.

It is worth noting that, $p_{\text {outlet }}$ controls the pressure drop along the runner cascade. The example of pressure distribution along the outer border of runner domain is shown in Fig. 3. The parameters are: $p_{\text {inlet }}=$ $8,000 \mathrm{~Pa}, p_{\text {outlet }}=-5,000 \mathrm{~Pa}$ and $-20,000 \mathrm{~Pa}, n_{b}=2.0$.

The influence of the pressure drop on the designed runner blades was thoroughly investigated by means of 2D and 3D models.

For 3D model, the rest of boundary conditions are formulated according to the version of turbulence model chosen for the computation. This is the matter of commercial code (ANSYS/Fluent 15) and therefore it will not be discussed here.

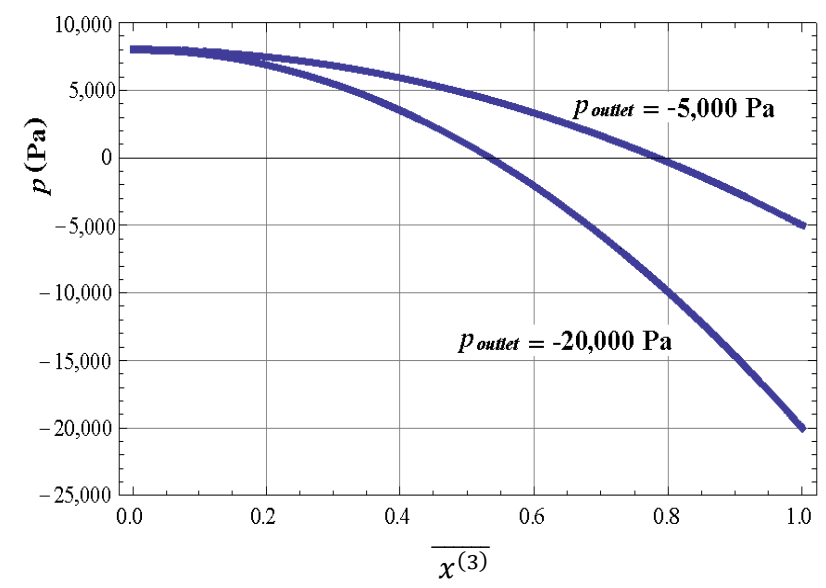

Fig. 3 Examples of pressure distribution at outer border of the runner domain for 2D model.

\section{Short Description of 2D Inverse Model}

The main points of algorithm of the inverse $2 \mathrm{D}$ model are described in Refs. [1-8]. Therefore the details of such approach will be omitted. Method of solution of momentum conservation equation leads to the determination of pressure field in domain of runner.

Having pressure determined the rest of parameters i.e., meridional and tangential components of velocity, respectively denoted as $U_{x^{(3)}}$ and $U_{x^{(2)}}$, can be computed from mass conservation equation:

$$
\left(1-\tau\left(x^{(1)}, x^{(3)}\right)\right) \rho U_{x^{(3)}} \frac{f\left|\frac{\partial f}{\partial x^{(1)}}\right|}{\sqrt{1+\left(\frac{\partial f}{\partial x^{(3)}}\right)^{2}}}=m\left(x^{(1)}\right)
$$

and energy conservation equation:

$$
\frac{1}{2}\left(U_{x^{(2)}}^{2}+U_{x^{(3)}}^{2}\right)-U_{x^{(2)}} U_{r o t}+\frac{p}{\rho}+\Pi+e=e_{c}\left(x^{(1)}\right)
$$

The following notations are used: $m\left(x^{(1)}\right)$-mass flow rate, $\tau\left(x^{(1)}, x^{(3)}\right)$ - blockage function takes into account the space occupied by the material of blades, $p$-pressure, $\rho$-density, $\quad U_{\text {rot }}$-circumferential velocity of runner, $e$ - internal energy, $e_{c}$ - total energy, $\Pi$-potential energy, $f$-radius which is stream function dependent on $x^{(1)}$ and $x^{(3)}$.

At runner inlet, along the leading edge, the values $m$ and $e_{c}$ are given from the boundary conditions. They are dependent on an $x^{(1)}$ coordinate.

Blockage function was chosen as below:

$\tau\left(x^{(1)}, x^{(3)}\right)=t_{1}\left(\overline{x^{(3)}}\right)^{t_{2}}\left(1-\overline{x^{(3)}}\right)^{t_{3}}\left(1-t_{4} x^{(1)}\right)$

Intuitively chosen parameters for blockage factor are: $t_{1}=0.2, t_{2}=0.5, t_{3}=1.0, t_{4}=-0.5$.

It generates the distribution of blockage function as it is shown in Fig. 4.

The arguments $x^{(1)}$ and $\overline{x^{(3)}}$ are within range $<0-1>$. The shape of profile generated by this blockage function is shown in Fig. 5.

The line crossing the profile in middle of thickness is the skeleton line which is determined as the trajectory of fluid element at outer contour of runner domain.

The suction side of profile is given by coordinates: 


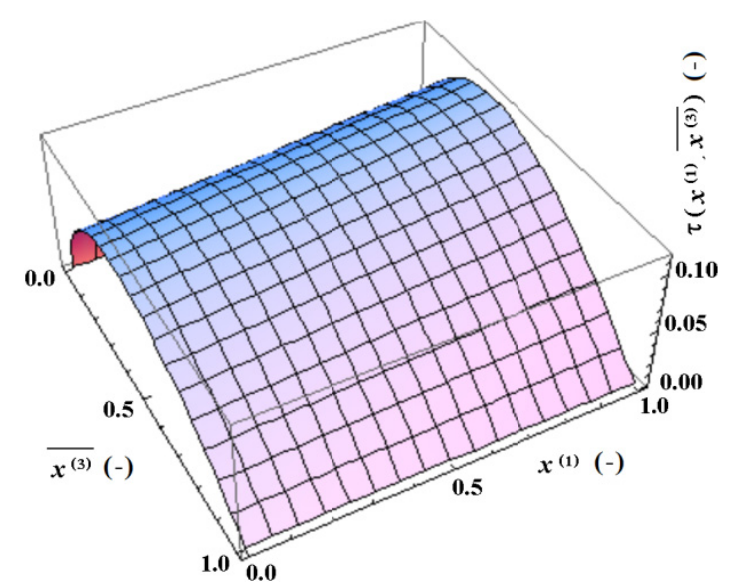

Fig. 4 Blockage function in the runner domain.

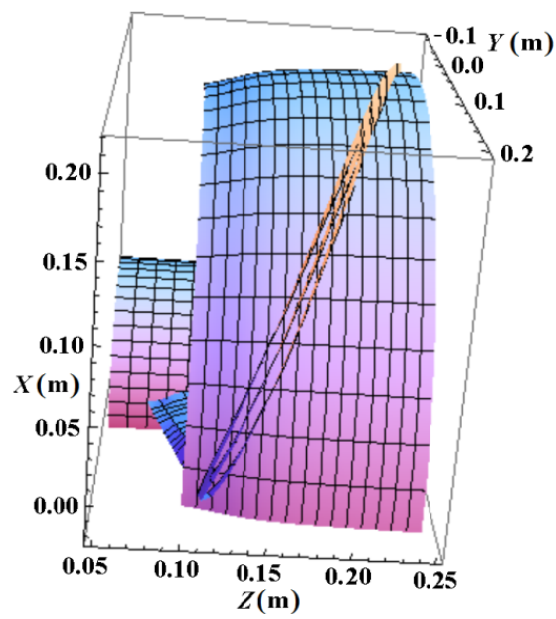

Fig. 5 The profile of runner at outer contour generated by means of a $2 \mathrm{D}$ inverse model.

$$
\begin{gathered}
x_{s}=r_{\text {outer }} \sin \left(x^{(2)}-2 \pi\left(1-t_{p}\right) \tau / z\right) \\
y_{s}=r_{\text {outer }} \cos \left(x^{(2)}-2 \pi\left(1-t_{p}\right) \tau / z\right) \\
z_{s}=r_{\text {outer }}
\end{gathered}
$$

and for the pressure side:

$$
\begin{gathered}
x_{p}=r_{\text {outer }} \sin \left(x^{(2)}+2 \pi t_{p} \tau / z\right) \\
y_{p}=r_{\text {outer }} \cos \left(x^{(2)}+2 \pi t_{p} \tau / z\right) \\
z_{p}=r_{\text {outer }}
\end{gathered}
$$

where, $z$ - the number of blades, $t_{p}$ - the division of thickness of profile at both sides of skeleton line.

In the energy conservation equation, the quantity $e=e\left(x^{(1)}, x^{(3)}\right)$ appears which represents the internal energy. From inlet to outlet of runner domain, this function ought to be determined in advance by means of a loss coefficient. It is a matter of intuition to prescribe the level of loss coefficient. In the present paper, authors propose the following form of loss coefficient distribution in runner domain for two cases different: (a) and (b):

$$
\begin{aligned}
\zeta\left(x^{(1)}, x^{(3)}\right)=( & w_{0} \\
& \left.+w_{1} x^{(1)}\right)\left(w_{2}+w_{3} x^{(1)}\right. \\
& \left.+w_{4}\left(x^{(1)}\right)^{2}\right)^{n_{l}}\left(\overline{x^{(3)}}\right)^{n_{s}}
\end{aligned}
$$

In case (a), parameters were chosen as follows:

$$
\begin{gathered}
w_{0}=0.017, w_{1}=0.0205, w_{2}=2.42842, \\
w_{3}=-3.36992, w_{4}=1.983, n_{l}=3.0, n_{s}=2.0(11 \mathrm{a})
\end{gathered}
$$

and in case (b), parameters were chosen as follows:

$$
\begin{gathered}
w_{0}=0.0061, w_{1}=0.00732, w_{2}=2.42842, \\
w_{3}=-3.36992, w_{4}=1.983, n_{l}=3.0, n_{s}=2.0
\end{gathered}
$$

They lead to the loss coefficient distribution shown for both cases in Fig. 6.

Then the increase of internal energy was computed in the reference of kinetic energy at the inlet of runner domain:

$$
\Delta e=\zeta\left(x^{(1)}, x^{(3)}\right)\left(\frac{U_{x}^{2}(2)+U_{x}^{2}(3)}{2}\right)_{\text {inlet }}
$$

The level of inlet kinetic energy was kept constant so it is possible to adjust the value of internal energy increase only by loss coefficient distribution.

\section{Some Results of 2D Computations}

The main goal of 2D inverse problem computation was to generate the geometry of the runner blades. After that, the generated blade geometry was examined by means of $3 \mathrm{D}$ computation model.

In order to diminish the number of cases in $2 \mathrm{D}$ computations, it was assumed the scheme computation presented in Table 1 below.

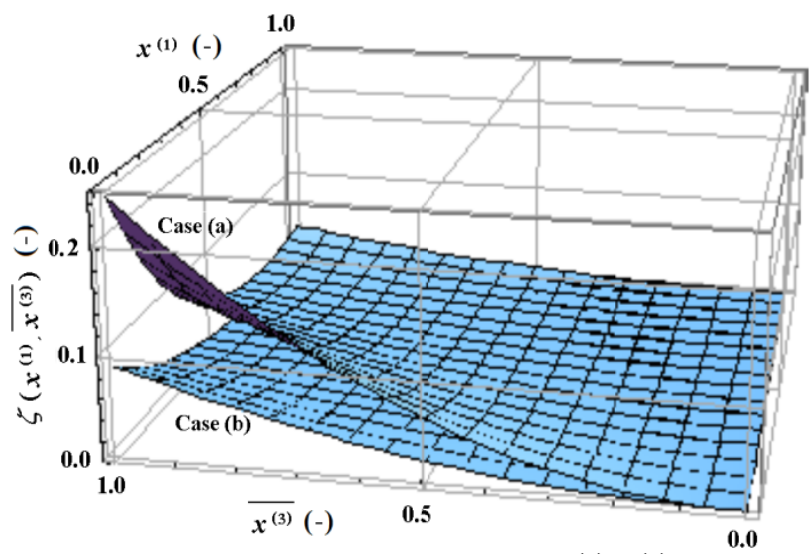

Fig. 6 Loss coefficient distribution $\zeta\left(x^{(1)}, x^{(3)}\right)$ for two analysed cases. 
Table 1 Variants of 2D model computations.

\begin{tabular}{|c|c|c|}
\hline \multirow[b]{2}{*}{$\begin{array}{l}\text { Pressure } p_{\text {outlet }} \text { at upper corner } \\
\text { of the runner blades (reference } \\
\text { parameter) }\end{array}$} & Convergent contour & Divergent contour \\
\hline & $\begin{array}{l}p_{\text {outlet }}=(-20,000)-5,000 \mathrm{~Pa} \\
(-5,000,-6,000,-7,000,-8,000,-11,000,-13,000, \\
-15,000,-20,000,-23,000,-28,000,-30,000)\end{array}$ & $\begin{array}{l}p_{\text {outlet }}=(-26,000)-5,000 \mathrm{~Pa} \\
(-5,000,-6,000,-7,111,-12,000,-18,000, \\
-23,426,-28,000,-26,000)\end{array}$ \\
\hline
\end{tabular}

The reference parameter $p_{\text {outlet }}$ will be plotted as an abscissa in the most figures presented below.

For convergent contour, two examples of geometry are shown in Fig. 7. For divergent contour, the similar geometries are shown in Fig. 8.

To compare the computed cases, the mean values of parameters averaged over the inlet and outlet surfaces were used.

The mean pressure drop along the runner blades versus $p_{\text {outlet }}$ is shown in Fig. 9. No difference in pressure drop is visible for both contours.

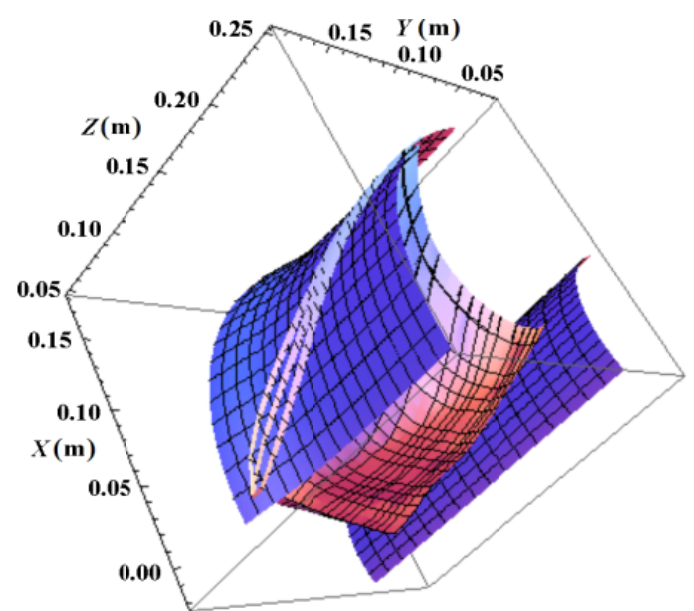

(a)

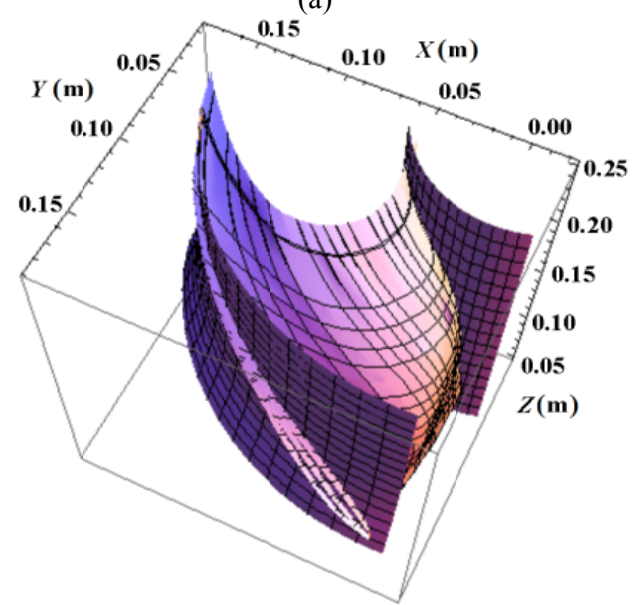

(b)

Fig. 7 Two examples of geometry for convergent contour ((a): $\left.p_{\text {outlet }}=-5,000 \mathrm{~Pa},(\mathrm{~b}): p_{\text {outlet }}=-20,000 \mathrm{~Pa}\right)$.

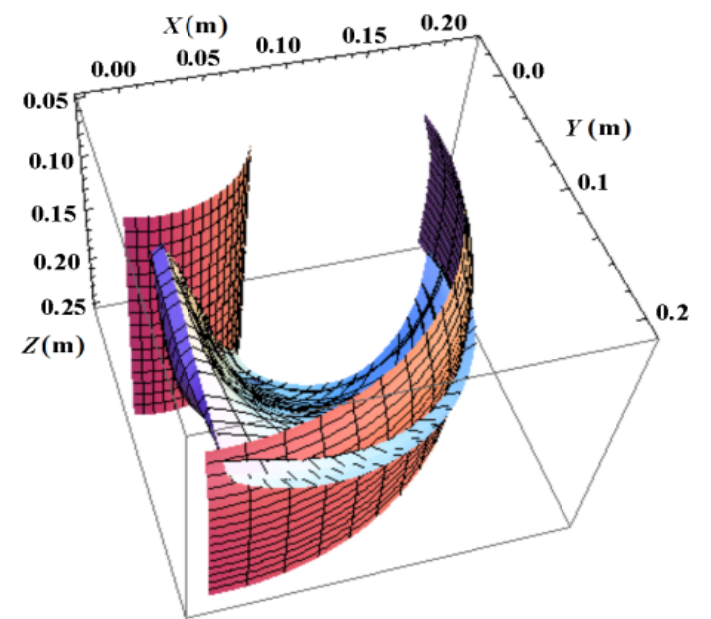

(a)

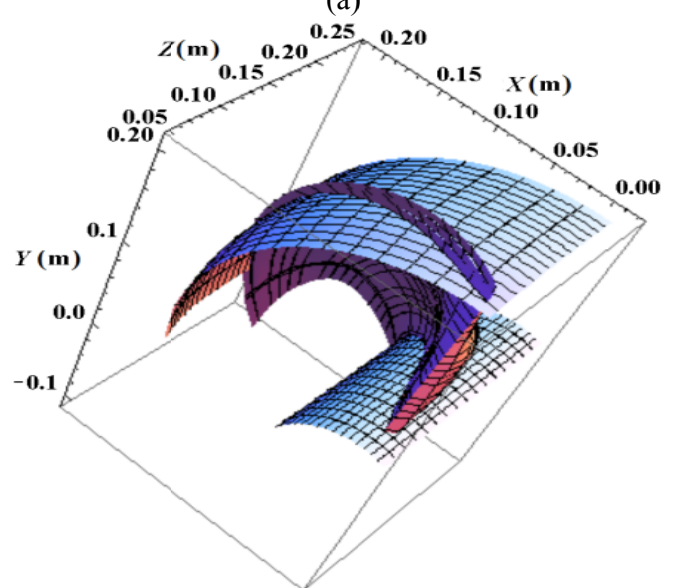

(b)

Fig. 8 Two examples of geometry for divergent contour ((a): $\left.p_{\text {outlet }}=-5,000 \mathrm{~Pa},(\mathrm{~b}): p_{\text {outlet }}=-20,000 \mathrm{~Pa}\right)$.

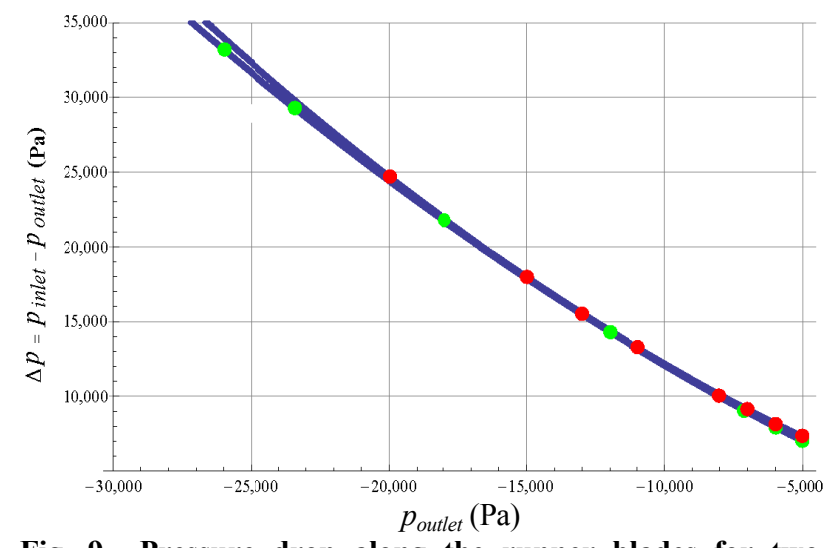

Fig. 9 Pressure drop along the runner blades for two contours (convergent and divergent). 


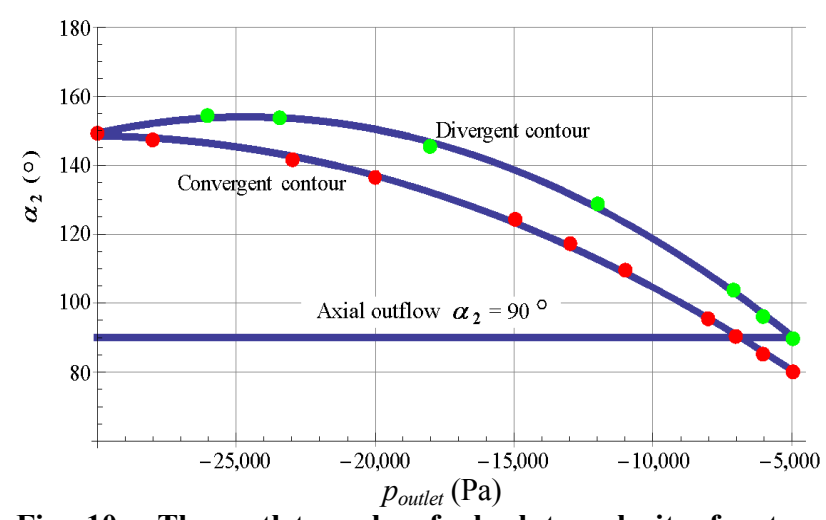

Fig. 10 The outlet angle of absolute velocity for two contours (convergent and divergent).

The outlet angle of absolute velocity is shown in Fig. 10.

The most interesting cases are those in which the outlet angle value $\alpha_{2}$ is close to $90^{\circ}$ what means axial outflow. The argument for the axial outflow comes from a discussion of energy equation. Let us define the energy at the inlet and outlet to runner (assuming that, all parameters are averaged), which are available to the conversion into mechanical energy in the runner domain:

$$
\begin{aligned}
& E_{\text {inlet }} \stackrel{\text { def }}{=} \frac{U_{x^{(2)}{ }_{\text {inlet }}}^{2}}{2}+\frac{U_{x^{(3)}{ }_{\text {inlet }}^{2}}^{2}}{2}+\frac{p_{\text {inlet }}}{\rho}+\Pi_{\text {inlet }} \\
& E_{\text {outlet }} \stackrel{\text { def }}{=} \frac{U_{x^{(2)} \text { outlet }}^{2}}{2}+\frac{U_{x^{(3)}}^{2} \text { outlet }}{2}+\frac{p_{\text {outlet }}}{\rho}+\Pi_{\text {outlet }}
\end{aligned}
$$

Thus, energy conservation equation along the runner domain can be written as:

$$
\begin{gathered}
E_{\text {inlet }}+e_{\text {inlet }}-\left(U_{x^{(2)}} U_{\text {rot }}\right)_{\text {inlet }}= \\
E_{\text {outlet }}+e_{\text {outlet }}-\left(U_{x^{(2)}} U_{\text {rot }}\right)_{\text {outlet }}
\end{gathered}
$$

Energy conservation equation can be rewritten as follows:

where:

$$
E_{\text {inlet }}=E_{\text {outlet }}+a+\Delta e
$$

$$
a=\left(U_{x^{(2)}} U_{\text {rot }}\right)_{\text {inlet }}-\left(U_{x^{(2)}} U_{\text {rot }}\right)_{\text {outlet }}
$$

is the amount of energy converted by the runner and

$$
\Delta e=e_{\text {outlet }}-e_{\text {inlet }}>0
$$

is the increase of internal energy due to dissipation and now can be presented in the form:

$$
\begin{aligned}
& E_{\text {inlet }}=\frac{U_{x^{(3)}{ }_{\text {outlet }}}^{2}}{2}+\frac{p_{\text {outlet }}}{\rho}+\Pi_{\text {outlet }}+a \\
& \overbrace{\Delta e+\frac{U_{x^{(2)} \text { outlet }}^{2}}{2}}^{\text {Lost energy (Le) }}
\end{aligned}
$$

The kinetic energy related to the meridional velocity component $U_{x^{(3)}{ }_{\text {outlet }}}^{2} / 2$ is tied up with mass conservation equation and cannot be minimized for the given mass flow rate. The kinetic energy of tangential velocity component $U_{x^{(2)}{ }_{\text {outlet }}}^{2} / 2$ can be treated as the part of lost energy $(L e)$. If there is no means to convert this amount of energy into pressure energy, it inevitably will be dissipated. Then, we can treat the sum:

$$
L e=\frac{U_{x}^{2}(2)}{2}+\Delta e
$$

as losses, which has to be minimized. This is the reason why behind the runner, the axial velocity is preferable. The existence of tangential velocity diminishes the amount of work converted by the runner. Fig. 11 shows the sum Le as a function of $p_{\text {outlet }}$.

On the basis of Fig. 11, the conclusion can be drawn that, the minimum Le places in the neighborhood of $p_{\text {outlet }}=-5,000 \mathrm{~Pa}$. This points out the geometry which can be considered as an advisable. Nevertheless, such conclusion should be checked by means of $3 \mathrm{D}$

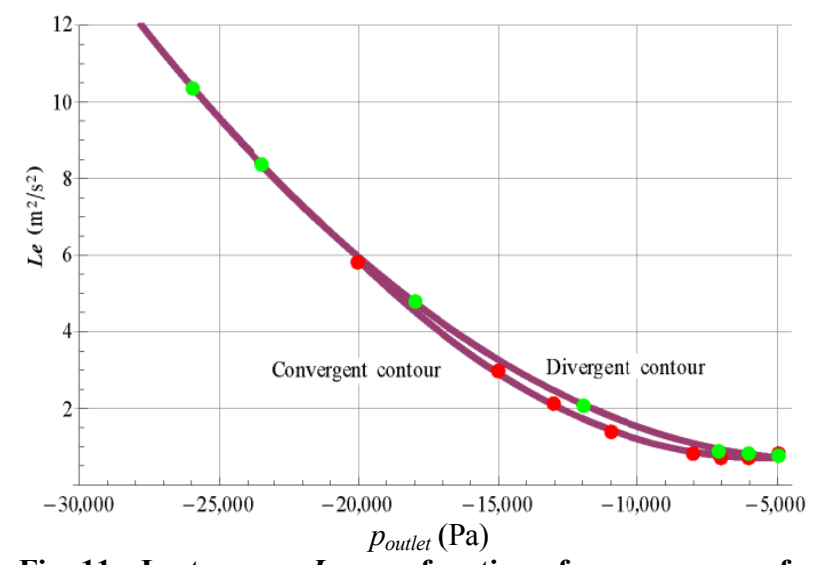

Fig. 11 Lost energy $L e$ as a function of pressure $p_{\text {outlet }}$ for two contours (convergent and divergent). 
computation. Two reasons will influence on the differences between 3D and 2D models:

(1) The finite number of blades which stands in opposition to infinite number of blades in 2D model;

(2) 3D computation provides more realistic estimation of dissipation effect than it is usually assumed in 2D computation.

Fig. 12 shows the level of dissipation energy introduced in 2D computation for two cases of loss coefficient distribution (a) and (b).

One can notice from Fig. 12 that, dissipated energy for two contours is of the same value. The loss coefficient identically distributed in the domain of runner for both contours leads to the same level of dissipation.

The energy equation is valid also for an arbitrary point of runner domain. One can rewrite this equation into the following form:

$$
\begin{aligned}
E_{\text {inlet }}=\frac{U_{x^{(2)}}^{2}}{2}+ & \frac{U_{x^{(3)}}^{2}}{2} \\
+ & \underbrace{\left(U_{x^{(2)}} U_{\text {rot }}\right)_{\text {inlet }}-\left(U_{x^{(2)}} U_{\text {rot }}\right)}_{a}+ \\
& +\underbrace{e-e_{\text {inlet }}}_{\Delta e}+\frac{p}{\rho}+\Pi
\end{aligned}
$$

Left hand side of above equation contains given values at the runner inlet. Right hand side can be treated as the quadratic equation for $U_{x^{(2)}}$ if the rest of parameters are given. Pressure field in runner domain can be computed according to the method described in Ref. [1]. The solution of above equation has the form:

$$
U_{x^{(2)}}=U_{\text {rot }} \mp \sqrt{\Delta}
$$

where, $\Delta$ is the discriminant of quadratic equation.

It is possible to have the situation when there is no real solution for $U_{x^{(2)}}$ due to negative discriminant. This may happen when the blockage factor and the increase of internal energy due to dissipation are too high. Then we may have the situation where at some points in runner domain no solution exists. The grids of points where the solution was sought for are shown in Fig. 13 for $p_{\text {outlet }}=-5,000 \mathrm{~Pa}$.

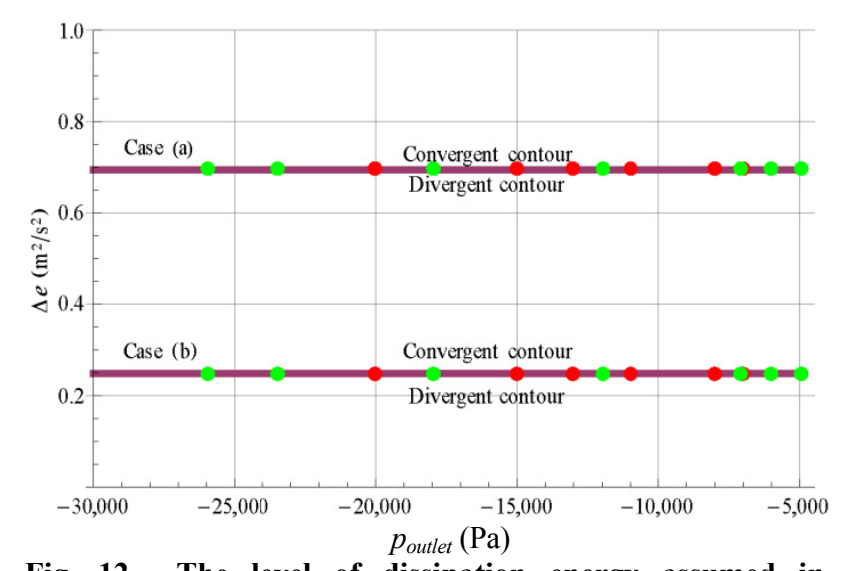

Fig. 12 The level of dissipation energy assumed in computation by means of $2 \mathrm{D}$ model.

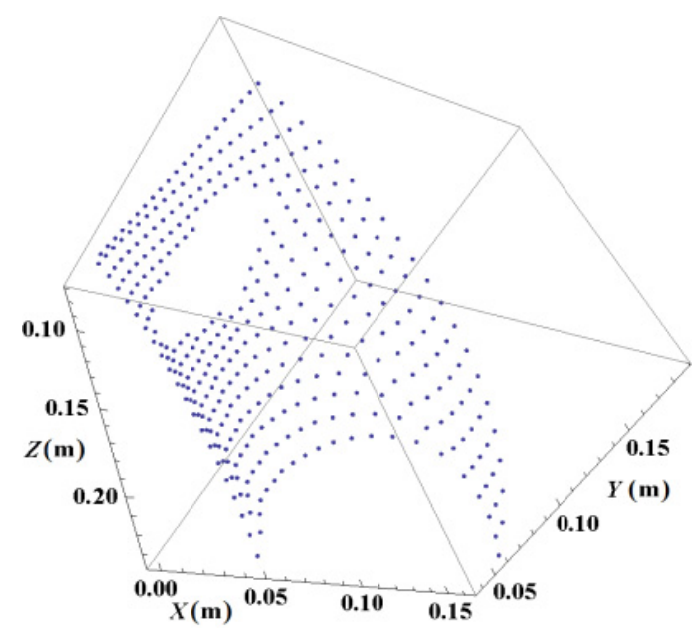

(a)

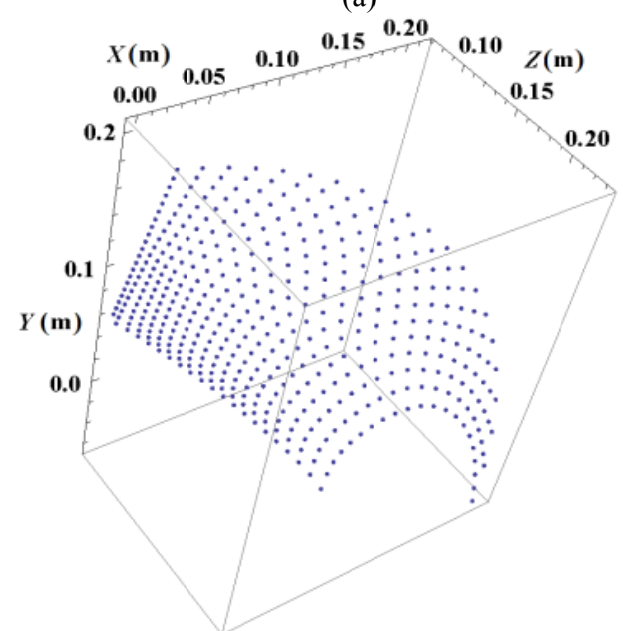

(b)

Fig. 13 (a) Convergent and (b) divergent blades obtained by means of 2D model for $p_{\text {outlet }}=-5,000 \mathrm{~Pa}$.

The Fig. 13a presents convergent case and Fig. 13b presents divergent case. In case of convergent contour, the empty region of blade ("white spot") close to the leading edge may be observed in which no solution for 
$U_{x^{(2)}}$ was obtained. In the case of divergent contour (Fig. 13b), the solution was obtained in the whole runner domain for comparable set of parameters.

In the convergent contour, the decrease of cross section due to convergence increased the meridional velocity $U_{x^{(3)}}$. The decrease of cross section manifests the increase of blockage factor. To fill up the "white spot" area, an additional assumption should be introduced. To create surface where no solution is obtained, geometrical extrapolation from the neighboring area may be applied or if $\frac{\sqrt{|\Delta|}}{U_{\text {rot }}} \ll 1$, the approximation $U_{x^{(2)}} \approx U_{\text {rot }}$ is justified. In this way, the geometry based on $2 \mathrm{D}$ inverse model for $3 \mathrm{D}$ computation may be obtained.

\section{The 3D Computation of Flow} Domain-Estimation of Internal Energy Increase by Means of Kinetic Dissipation Energy Value

The 3D computation with version of turbulence model $k-\varepsilon$ provides with distribution of kinetic dissipation energy $\varepsilon$ in runner domain. In order to estimate the increase of the internal energy, due to turbulence let us examine two relations. The first one is the relation between entropy production $s_{m}$ and rate of dissipation $\varepsilon\left(\mathrm{m}^{2} / \mathrm{s}^{3}\right)$ :

$$
\varepsilon=\frac{T s_{m}}{\rho}
$$

where, $T$-absolute temperature, $\rho$ - density.

The second relation is the energy conservation equation written for internal energy:

$$
\rho \frac{\mathrm{d} e}{\mathrm{~d} t}=T s_{m}+\frac{p}{\rho} \frac{\mathrm{d} \rho}{\mathrm{d} t}+\lambda \Delta T
$$

One can simplify the above equation for incompressible fluid and neglecting the heat conduction. Combining these equations it can be written:

$$
\frac{\mathrm{d} e}{\mathrm{~d} t}=\varepsilon
$$

and integrating the increase of internal energy $\Delta e$ $\left(\mathrm{m}^{2} / \mathrm{s}^{2}\right)$ is estimated as follows:

$$
\begin{gathered}
\Delta e=\int_{e_{1}}^{e_{2}} \mathrm{~d} e=e_{2}-e_{1}=\int_{t_{1}}^{t_{2}} \varepsilon \mathrm{d} t=\bar{\varepsilon} \int_{t_{1}}^{t_{2}} \mathrm{~d} t= \\
\bar{\varepsilon}\left(t_{2}-t_{1}\right)=\bar{\varepsilon} \Delta t
\end{gathered}
$$

The mean value of $\bar{\varepsilon}$ let us estimate as:

$$
\bar{\varepsilon} \cong \frac{\varepsilon_{\text {inlet }}+\varepsilon_{\text {outlet }}}{2}
$$

The interval of time $\Delta t$ is related to the trajectory of representative fluid element which passes the runner domain. As a representative length $\Delta l_{\text {axial }}$, we can choose the axial length of the runner at mean diameter and mean value of axial velocity:

$$
\overline{U_{\text {axlal }}} \cong \frac{U_{\text {axial inlet }}+U_{\text {axial outlet }}}{2}
$$

then one can estimate:

$$
\Delta t \cong \frac{\Delta l_{\text {axial }}}{\overline{U_{\text {axial }}}}
$$

The foregoing considerations formulate the estimation of the increase of internal energy due to turbulent dissipation, which is the prevailing factor of dissipation.

\section{Some Results of 3D Computations}

Fig. 14 shows the level of internal energy increase due to dissipation according to the results of $3 \mathrm{D}$ computation by means of turbulence model $k-\varepsilon$.

The level of energy dissipation assumed for 2D computation, as it is shown in Fig. 12, was overestimated for the case (a) compared to the values in Fig. 14. The case (b) shows a good guess comparing with the $3 \mathrm{D}$ results.

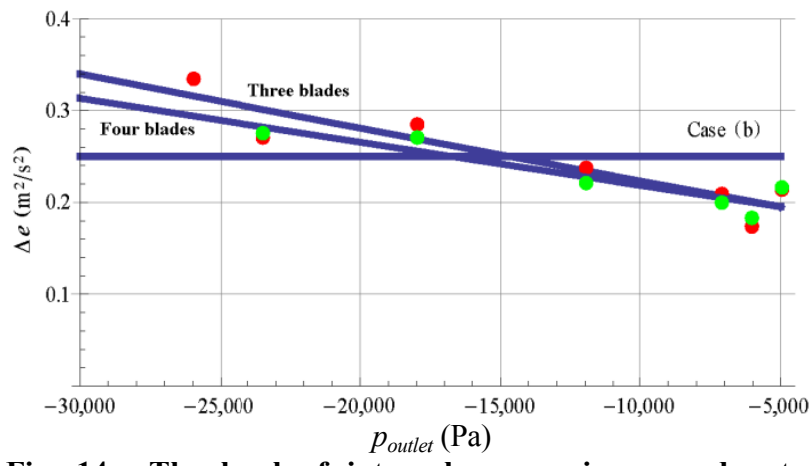

Fig. 14 The level of internal energy increase due to dissipation computed by means of turbulence model $k-\varepsilon$ (three and four blades runner for divergent contour). 
The difference of $\Delta e$ between three and four blades in runner is negligible, covered by the scatter of numerical results. The slight increase of dissipation for lower values of $p_{\text {outlet }}$ can be explained by increasing of the profiles length, as it can be seen in Figs. 7 and 8.

The sum of lost energy Le from 3D computation is shown in Fig. 15. Comparing to data in Fig. 12, it can be concluded that, the minimum is shifted to the left, giving the recommended solution at $p_{\text {outlet }}=-23,600 \mathrm{~Pa}$ for three blades and $p_{\text {outlet }}=-16,150 \mathrm{~Pa}$ for four blades.

Fig. 16 shows the averaged pressure drop along 3-blade runner domain. Comparing to the values of pressure at the inlet of the runner, one can notice that, outlet pressure is closer to zero. This is in accordance to

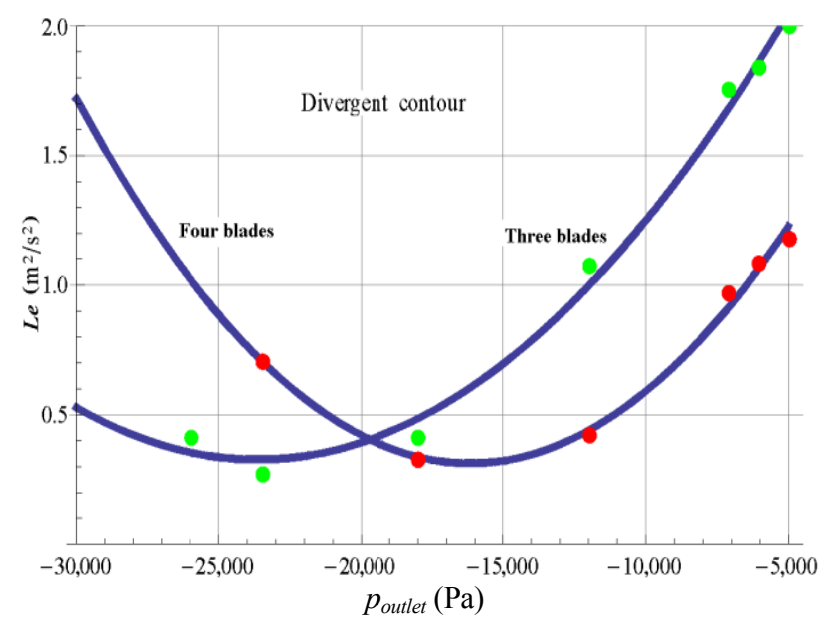

(a)

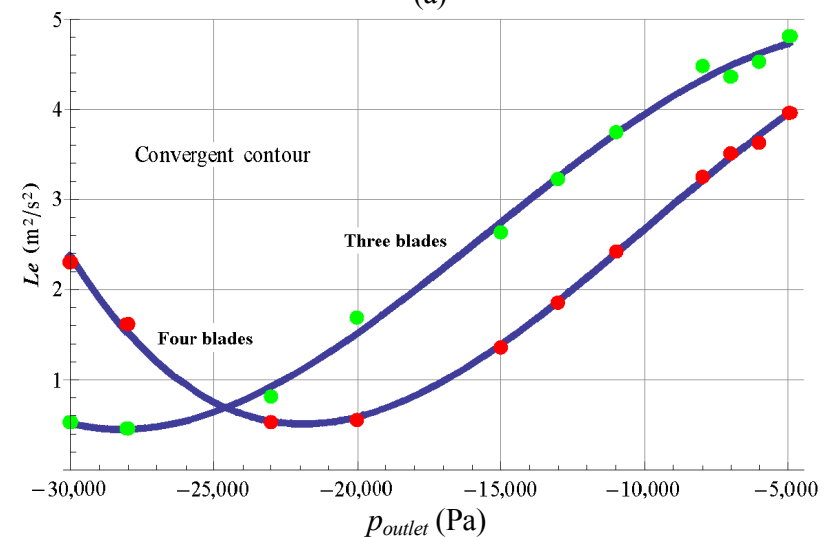

(b)

Fig. 15 Sum of $L e$ energy showing the minimum for three and four blades for (a) divergent contour and (b) convergent contour.

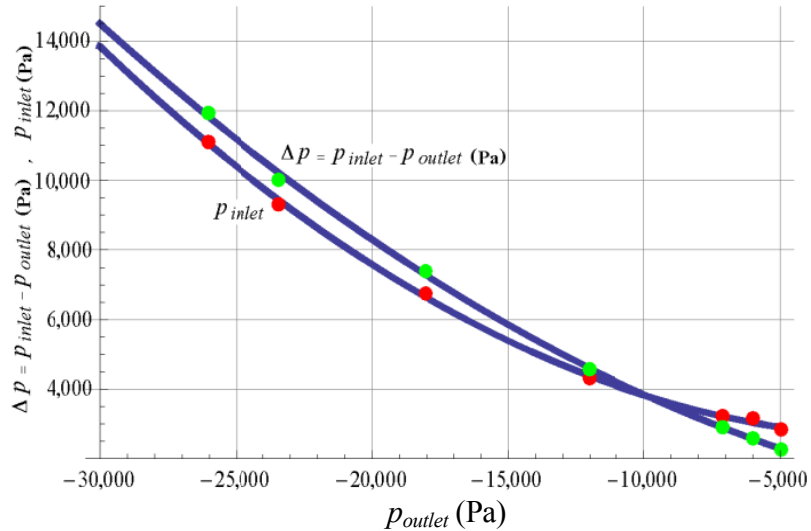

Fig. 16 Distribution of pressure $p_{\text {inlet }}$ at inlet to runner domain and pressure drop $\Delta p$ along the runner blades (3-blade runner with divergent contour).

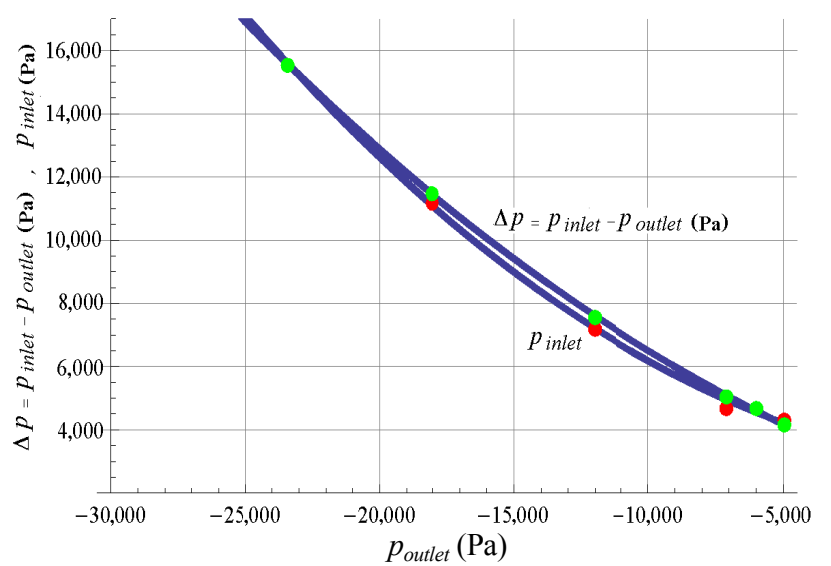

Fig. 17 Distribution of pressure $p_{\text {inlet }}$ at inlet to runner domain and pressure drop $\Delta p$ along the runner blades (4-blade runner with divergent contour).

the boundary condition for pressure in computation of 3D model.

Comparing the values in Fig. 16 to data in Fig. 10, it became evident that, in 2D model, we have a higher pressure drop due to the fact of infinite number of blades. For given mass flow rate, the cascade is more "dense", the higher pressure drop is obtained as it can be seen comparing Fig. 16 to Fig. 17 for two different numbers of runner blades ( 3 and 4-blade runner). It must be emphasized that, velocity conditions at the inlet to the runner was kept at the same level and the same was also the reference pressure behind the runner. In Fig. 18, the pressure drop for divergent contour and 3 and 4-blade runner is confronted. Table 2 presents loss energy, pressure at outlet and pressure difference for 3 and 4-blade runners. 


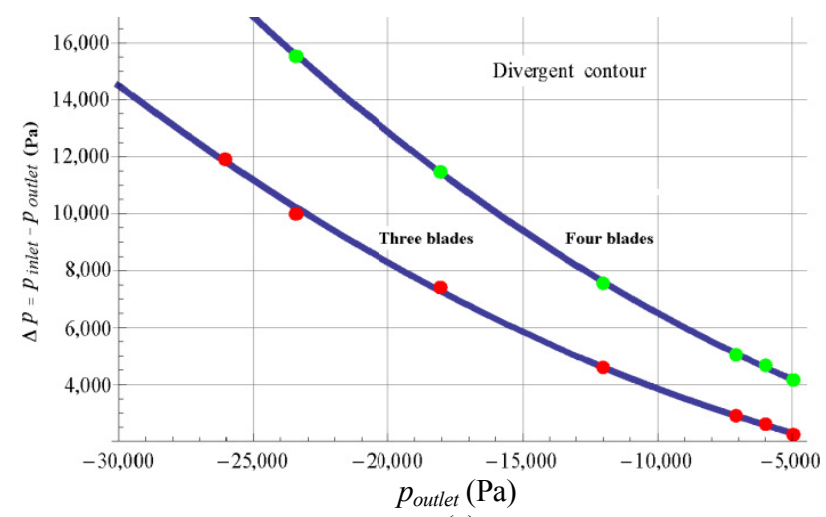

(a)

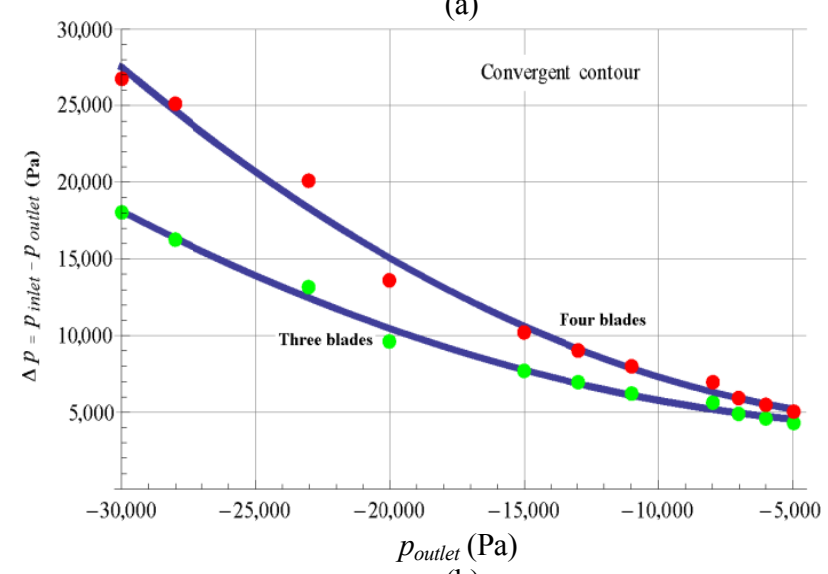

(b)

Fig. 18 Comparison of pressure drops for two different blade numbers of the runner with (a) divergent contour and (b) convergent contour.

Table 2 Comparison of 3D computations results for three and four blades runners.

\begin{tabular}{lllll}
\hline \multirow{2}{*}{ Parameters } & \multicolumn{2}{l}{ Divergent contour } & \multicolumn{2}{l}{ Convergent contour } \\
\cline { 2 - 5 } & $\begin{array}{l}\text { Three } \\
\text { blades }\end{array}$ & $\begin{array}{l}\text { Four } \\
\text { blades }\end{array}$ & $\begin{array}{l}\text { Three } \\
\text { blades }\end{array}$ & $\begin{array}{l}\text { Four } \\
\text { blades }\end{array}$ \\
\hline$L e_{\text {min }}\left(\mathrm{m}^{2} / \mathrm{s}^{2}\right)$ & 0.3267 & 0.3121 & 0.4514 & 0.5116 \\
$p_{\text {outlet min }}(\mathrm{Pa})$ & $-23,637$ & $-16,157$ & $-28,235$ & $-21,888$ \\
$\Delta p_{\text {stat }}(\mathrm{Pa})$ & 10,345 & 10,155 & 16,547 & 17,045 \\
& $\approx 10,000$ & $\approx 10,000$ & $\approx 17,000$ & $\approx 17,000$ \\
\hline
\end{tabular}

\section{Conclusions}

(1) $2 \mathrm{D}$ inverse method is effective to obtain the geometry of blades for the runner;

(2) 3D computation for two differently shaped runners shows that, from dissipation point of view, divergent runner is more effective that convergent one;

(3) The increase of the draft tube load for convergent runner may change the final decision concerning the channel shaping. Especially in the case when maximum power output (not efficiency) is the main target. This problem exceeds the volume of the present paper.

\section{Acknowledgments}

The work was supported by the Polish National Science Centre under grant no. 6694/B/T02/2011/40 for The Szewalski Institute of Fluid-Flow Machinery in Gdansk, Polish Academy of Sciences.

\section{References}

[1] Puzyrewski, R. 2005. Two Dimensional Inverse Method of Turbomachinery Stage Design, Developments in Mechanical Engineering. Gdansk: Gdansk University of Technology Publishers, 57-72. ISBN 83-7348-113-3.

[2] Puzyrewski, R., and Krzemianowski, Z. 2014. "2D Model of Guide Vane for Low Head Hydraulic Turbine: Analytical and Numerical Solution of Inverse Problem." Journal of Mechanical Engineering and Automation 4 (3): 195-202.

[3] Puzyrewski, R., and Krzemianowski, Z. 2015. "Two Concepts of Guide Vane Profile Design for a Low Head Hydraulic Turbine." Journal of Mechanical Engineering and Automation 5 (4): 201-9.

[4] Krzemianowski, Z., and Puzyrewski, R. 2014. "3D Computations of Flow Field in a Guide Vane Blading Designed by Means of 2D Model for a Low Head Hydraulic Turbine." Journal of Physics: Conference Series 530 (1): 1-8. doi: 10.1088/1742-6596/530/1/012031, ISSN 1742-6596 (Online).

[5] Puzyrewski, R., and Krzemianowski, Z. 2013. "Impact of Blade Quality on Maximum Efficiency of Low Head Water Turbine." Journal of Mechanics and Mechanical Engineering 17 (3): 269-84. ISSN 1428-1511.

[6] Puzyrewski, R. 1998. Lectures on Theory of Turbomachinery Stage-Two Dimensional Model (2D). Gdansk: Gdansk University of Technology.

[7] Puzyrewski, R., and Namieśnik, K. 1996. "Comparison between Conical and Paraboloidal Turbine Stages: Inverse Problem." Presented at the 3rd International Symposium on Experimental and Computational Aerothermodynamics of Internal Flows (ISAIF), Beijing, China.

[8] Flaszyński, P. 2002. "Comparison of Two Dimensional and Three Dimensional Models Based on Turbomachinery Flow Passages." Ph.D. thesis, Gdansk University of Technology. 